\title{
NOVEL DEPLOYMENT MECHANISM FOR CONVENTIONAL SOLAR ARRAY ENHANCEMENT
}

\author{
Paul A. Hodgetts ${ }^{(1)}$, Jean-Paul Collette ${ }^{(2)}$, Don de Wilde ${ }^{(3)}$ \\ (I)ESA/ESTEC, Keplerlaan 1, 2200 AG Noordwijk, The Netherlands, Email: Paul.Hodgetts@esa.int \\ (2)WalOpt, Avenue E. Ysaye 44, 4053 Embourg, Belgium,Email: jpcollette@walopt.com \\ (3) ESA/ESTEC, Keplerlaan 1, 2200 AG Noordwijk, The Netherlands, Email: Don.de.Wilde@esa.int
}

\begin{abstract}
A novel mechanism is described, by which flexible blankets could be deployed from existing solar panel designs. These blankets could be covered with flexible cells, or they could be reflective films to form a concentrator array. Either way, the performance of an existing array design could be enhanced.
\end{abstract}

\section{INTRODUCTION}

A conventional solar array comprises a backbone of rigid panels and a yoke, fixed in deployed configuration by lockable hinges. The performance of such an array $(\mathrm{W} / \mathrm{kg}$ ) could be enhanced in one of two ways:

- Lightweight blankets could be extended from either side of each rigid panel, equipped with flexible cells. When deployed, these blankets would lie in the same plane as the rigid panels. The additional cell area would increase the power output of the array, while not significantly increasing its total mass.

- Reflective membranes could be deployed from either side of each rigid panel, inclined at $60^{\circ}$ with respect to the plane of the respective panel. In this way, a reflective trough would be formed, thereby increasing the incident light on the existing cells of the rigid panels.

In either case, in stowed configuration, these blankets or foils would have to be wrapped around a cylindrical drum, positioned at either side of each panel.

These would have to be released and unwound in orbit, in such a way that their deployment would not inhibit the deployment of the rigid panel assembly.

A deployment mechanism, which fulfils these requirements, has been invented by the author (Patent No. WO 2015/062650 A1) the description of which is the subject of this paper.

\section{MECHANISM DESCRIPTION}

The principle of the mechanism is based on a crossed tether, wrapped around two wheels, forming a figure of eight. Attached to the tether are slender rods which are forced out laterally, when the wheels rotate and the tether is drawn.

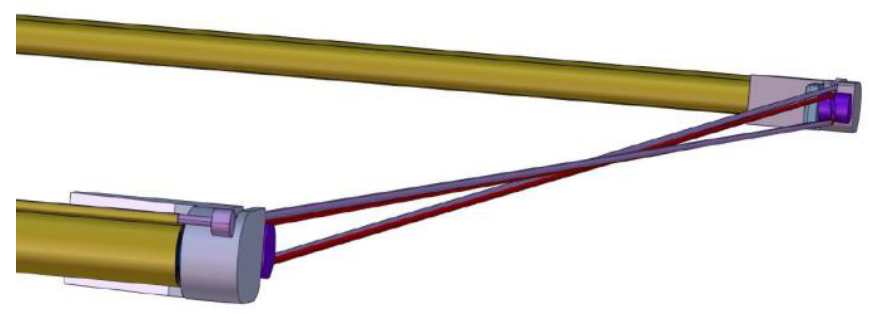

Figure 1. Core of Deployment Mechanism Showing Crossed Tether and Slender Rods

At the opposite end of the panel, an identical mechanism exists with a crossed tether and a pair of slender rods.

Attached to the ends of these slender rods are spreader bars, running the full length of the panel, one on either panel side. To each spreader bar, a blanket or reflective film is attached, which is wrapped around a cylindrical drum when stowed, with the spreader bars positioned adjacently.

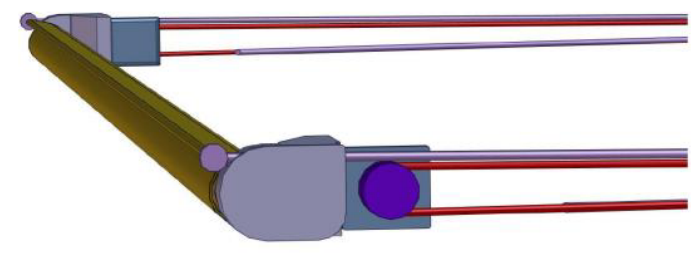

Figure 2. Slender Rods, Spreader Bar and Blanket/Reflective Foil in Stowed Configuration 
The complete assembly of two tethers, four slender rods, two spreader bars and two cylindrical drums, forms a rectangular shape.

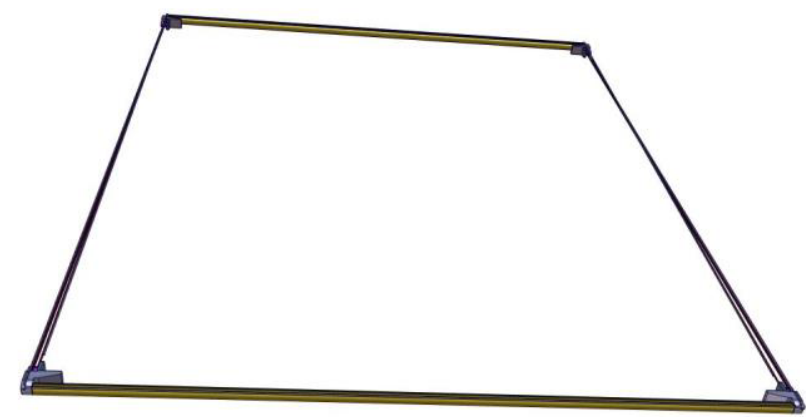

Figure 3. Deployment Mechanism and Blanket/Foil Assembly

The assembly fits around a conventional panel: the rods are accommodated in the edge member profile of the panel substrates, to which the panel hinges are attached.

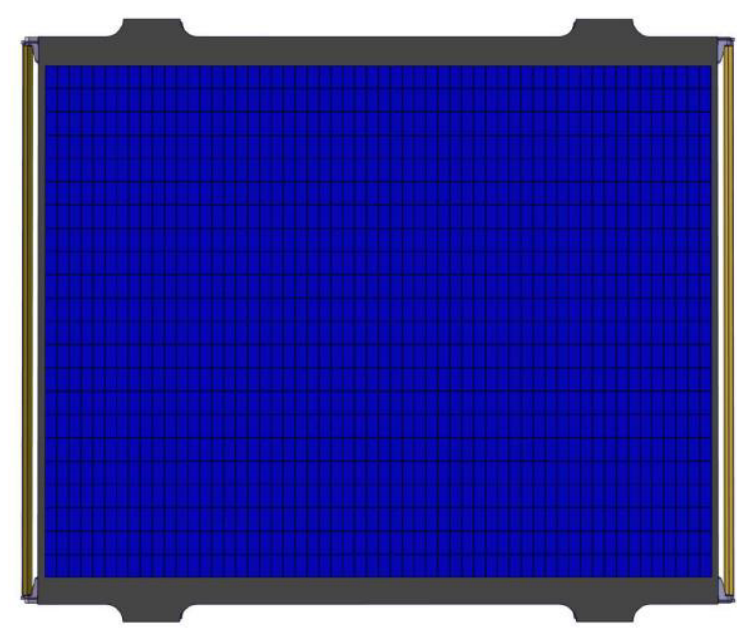

Figure 4. Accommodation of Deployment Mechanism and Blanket/Foil Assembly

The motion of the tethers is suppressed, until all panel hinges, on either side of a given panel, latch in orbit.

With the release of the tether, the rods are drawn by rotational springs connected to the pulley wheels. The motion of each mechanism, on either side of a given panel, is synchronised by the blanket/foil, as it is unwound from the cylindrical drums.

The slender rods are guided by a slot in the cylindrical drum support housing, which also provides a hinge location for the wheels, around which the tether is wound.

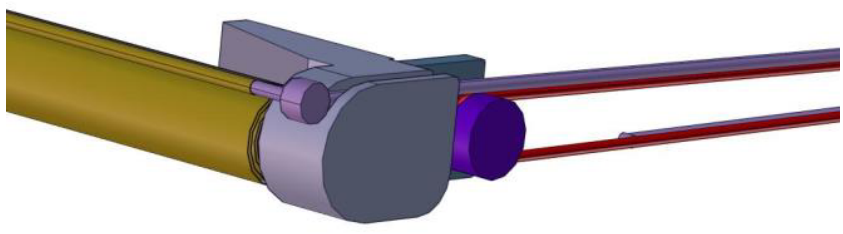

Figure 5. Cylindrical Drum Support Housing and Guidance Slot

At the end of deployment, the ends of these rods are forced into a recess, where they lodge.

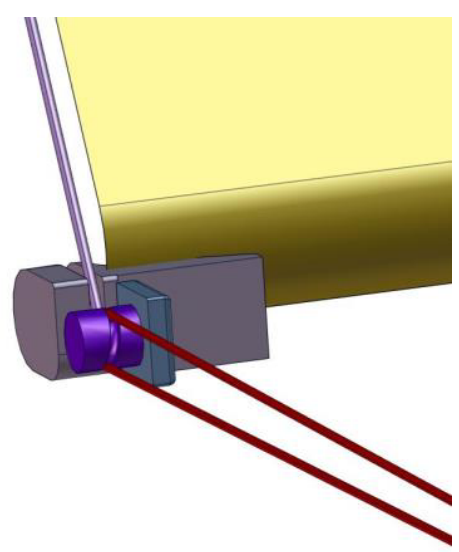

Figure 6. Slender Rod, Lodged in Recess

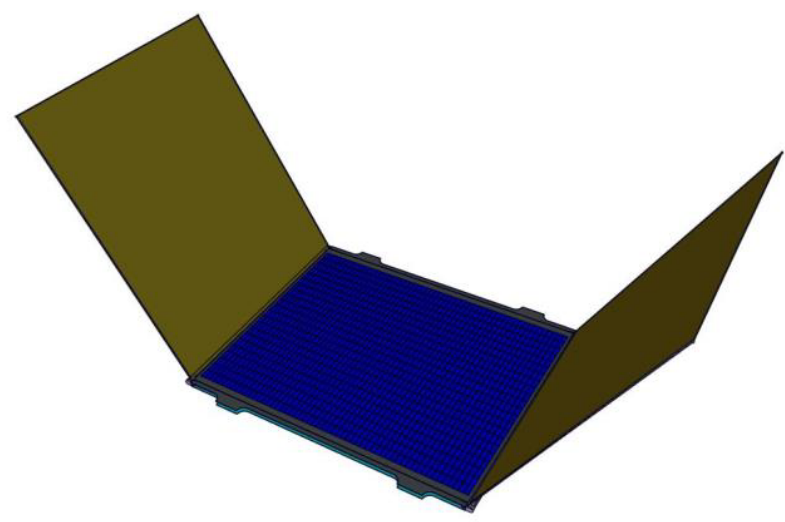

Figure 7. Reflective Foils in Fully Deployed Configuration 
The dimensions of the recess are such that, in the case of a reflective foil, these become inclined at 60 degrees with respect to the plane of the solar panel (Figure 7) thereby forming a reflective trough.

In the case of cell covered flexible blankets, the recess is such that the blankets would end up in a near flat orientation, with respect to the rigid panels.

\section{MECHANISM KINEMATICS}

In the case of the mechanism deploying a reflective film as part of a concentrator array, as the end of the slender rod rotates around the tether wheel (Figure 8) it subtends an angle $\theta$ with respect to its initial position, referred to as the mechanism deployment angle. The resulting inclination of the slender $\operatorname{rod} \psi$ is the foil inclination angle.

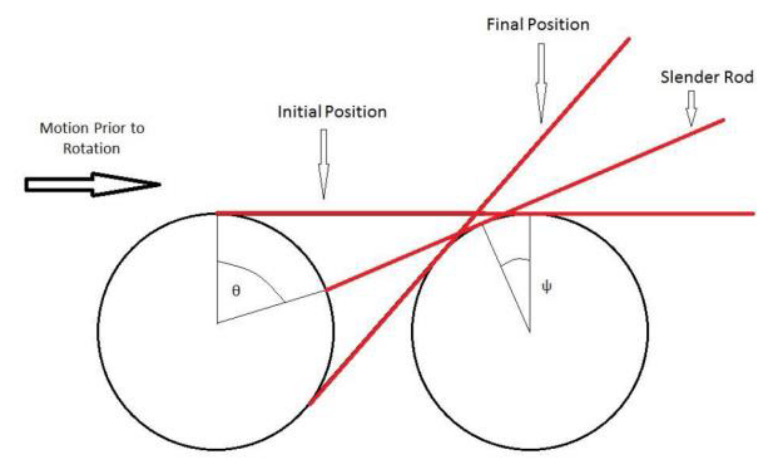

Figure 8. Schematic of Mechanism Showing Initial and Final Positions of Slender Rod

The kinematic relationship between these angles has been calculated (Figure 9). As can be seen, the foil inclination angle increases most rapidly with respect to mechanism deployment angle during mid-deployment.

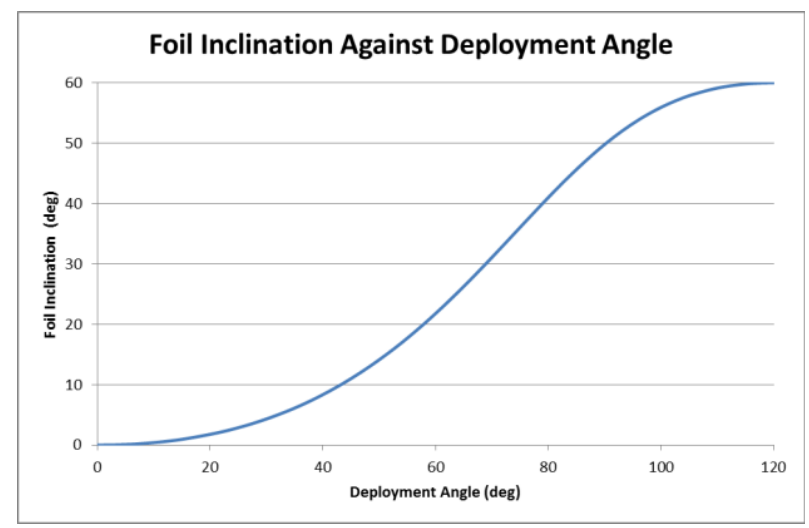

Figure 9. Kinematic Relationship between Foil Inclination Angle and Mechanism Deployment Angle
At the start and end of deployment, the slope is zero.

\section{DEPLOYMENT CHARACTERISTICS}

Since the mechanism is released the moment the panel hinges of a given panel latch, in the case of a multipanel array, blanket/foil deployment may take place while other panels are still in motion. Any jolts imposed on the wing, due to the blanket foil deployment, therefore need to be kept to a minimum.

As the mechanism involves two slender rod/spreader bar assemblies, which move simultaneously in opposite lateral directions, their inertial loads counteract one another, thereby generating a near zero in-plane impulse.

In the case of the reflective foil application, where significant out-of-plane motion exists, the kinematics show that as the slender rods start to rotate, much of their translational kinetic energy will be transferred into rotational kinetic energy. This will result in a rapid translational deceleration of the slender rod/spreader bar assemblies.

Precisely how the rods and reflective foils reach their final $60^{\circ}$ orientation will depend on frictional losses in the mechanism and the extent to which elastic energy is stored in the rods as they flex. While this is beyond the scope of the present study, expectations are that a soft end latch is achievable by means of tuning the frictional parameters via latch design and material selection.

\section{FUTURE DEVELOPMENT}

This deployment scheme is currently being further investigated by WalOpt, based in Liège (Belgium) as part of a feasibility study of concentrator solar arrays.

Multi-body dynamic analyses of the mechanism are being performed, based on panel dimensions typical of a telecom satellite array. In addition, a small scale breadboard model is to be manufactured, to assess its functionality. Particular attention will be paid to the suppression of wrinkles in the reflective film, since these are known to generate a non-uniform illumination of the solar cells.

\section{REFERENCES}

[1] "Small Satellite REIMEI for Auroral Observations", H. Saito, et al., Proceedings of the $61^{\text {st }}$ International Astronautica Congress, 2011. 